http://bjas.journals.ekb.eg

\title{
Current concept review of Sever's disease in paediatric age group
}

G.A.Hosny, M.I.Al-Ashhab, M.A.Moeselhy and M.M.Abdrabboh

Orthopaedic Surgery, Dept., Faculty of Medicine, Benha Univ., Benha, Egypt

E-mail:mohamed146@gmail.com

\section{Abstract}

Background: Sever's disease is directly associated with excessive usage of the heel bone and tendons. This may occur from any sports involving a lot of heel movement. The commencement of new sport or the beginning of new season may be linked with it. Children through puberty are particularly at danger since the heel bone is growing faster than the leg. The objective of this research was to conduct a current concept study on severity in the paediatric age range. Methods: This is an article of review, Searches in MEDLINE and Embase, Pubmed and CINAHL Plus were carried out within the same date period as follows: "Sever's disease; paediatric; orthopaedics." The most obvious sign of severe illness is heel discomfort, typically exacerbated by physical activity like walking, jogging, or skipping. Sometimes the discomfort may be so intense that it limps and affects physical performance in athletics. Sever's disease is most often clinically diagnosed and many doctors feel that radiographic assessment is unnecessary, however if calcaneal apophysitis is not identified without X-rays, an injury needing more severe treatment may be overlooked. Edematous alterations in calcaneal apophysis may occur via MRI, which may spread to neighbouring calcaneal tuberosity, The research indicates that most people with the Sever illness are able to return to sport in a few weeks to two months with appropriate management.

Keywords: Sever's disease, pediatric, Orthopedics.

\section{Introduction}

Sever's illness is named after James Warren Severe (1878-1964) who was described by an American orthopaedic specialist in 1912. Sever's disease 'also known as calcane apophysitis' involves inflammation of the heel's growth plate in developing young children [1].

Sever's illness is directly linked to bone and tendon overuse in the heel. This may occur from any sports involving a lot of heel movement. Starting a new sport may be linked [1].

Or the beginning of the new season. Kids who are in adolescence are particularly at danger because the heel bone develops faster than the leg. Too much weight may be caused on the heel as can excessive traction, as bones and tendons continue to grow. In general, both heels are involved in more than half of patients [2].

The most obvious sign of severe illness is heel discomfort, typically exacerbated by physical activity like walking, jogging, or skipping. The discomfort is located on the back and plantar of the heel above the calcane apophysis. Sometimes the discomfort may be so intense that it limps and interferes with sports' physical performance [2].

The external look of the heel is nearly always normal and there are no indications of local diseases such as edoema and erythema (roughness). The primary diagnostic technique is discomfort on the medial-lateral calcane compression on the region of development plate, known as the squeeze test. The diagnosis of severe illness is thus mainly clinical[1].

Sever's disease is diagnosed clinically most frequently, and radiography is considered to be unnecessary by many doctors, although a lesion needing more severe therapy may be overlooked if a diagnosis of calcaneal apophysitis is established without $\mathrm{x}$-rays.
Foot radiographs are typically normal, and without lack of clinical information, radiological diagnosis of calcaneal apophysitis has not been reliable [3]. MRI may indicate edoema alterations in calcaneal apophysis that may extend to the surrounding calcaneal tuberosity [2].

The purpose of this research is to carry out an up-to-date concept evaluation on severe illness in children.

\section{Methods}

This is a review article, The search was performed in MEDLINE, Embase, Pubmed and CINAHL Plus in the same date range with the following mediacl terms: "Sever's disease; pediatric; Orthopedics.", including articles from 2000 to 2021, Excluded articles from review are those of langauge other than English

\section{Results \\ Sever's Disease Defined}

Sever's disease is an inflammation of the calcaneal apophysis. The calcaneal apophysis is a cartilaginous growth center onto which the Achilles tendon in-serts. adding that Sever's disease is a traction epiphysitis as opposed to other forms of inflamma-tion at this site, such as bruising or infection. [4]

The calcaneal apophysis is the posterior as-pect of the calcaneus (see Figure 1), where the Achilles tendon inserts. The physis is the open growth plate. The calcaneus growth plate does not close until at least 14 years of age. Until that time, new bone is forming at the calcaneal physis. Irritation of this growth plate of the calcaneus creates stress at the growth plate, resulting in inflammation. The primary theory for Sever's injury is mechani-cal overuse due to repetitive impact pressure and shear stresses to this open growth plate of the calcaneus. 


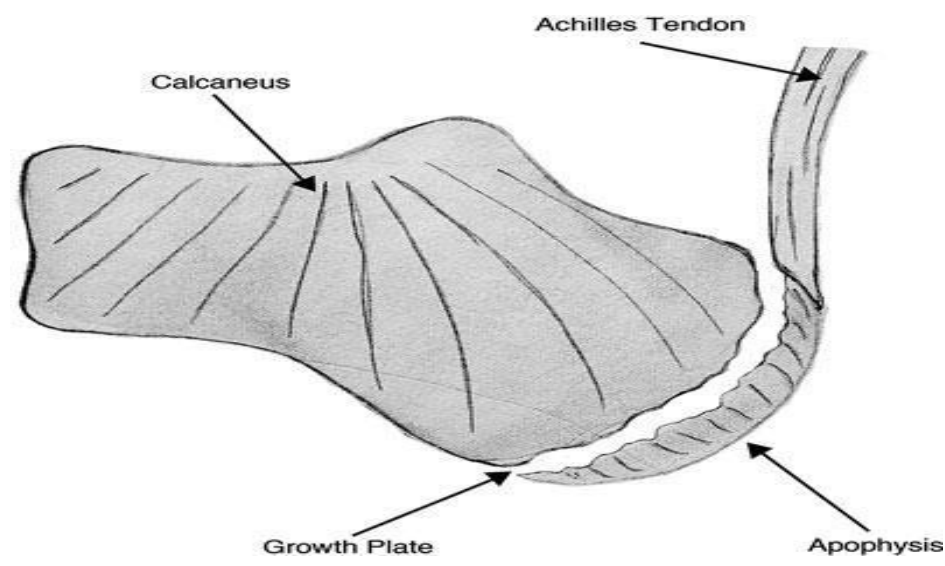

Fig. (1) Calcaneal apophysis is located posteriorto calcaneus. Calcaneal apophysis is the insertion site of the Achilles tendon. Illustration is the origi-nal artwork by Nicole Eaton. [4]

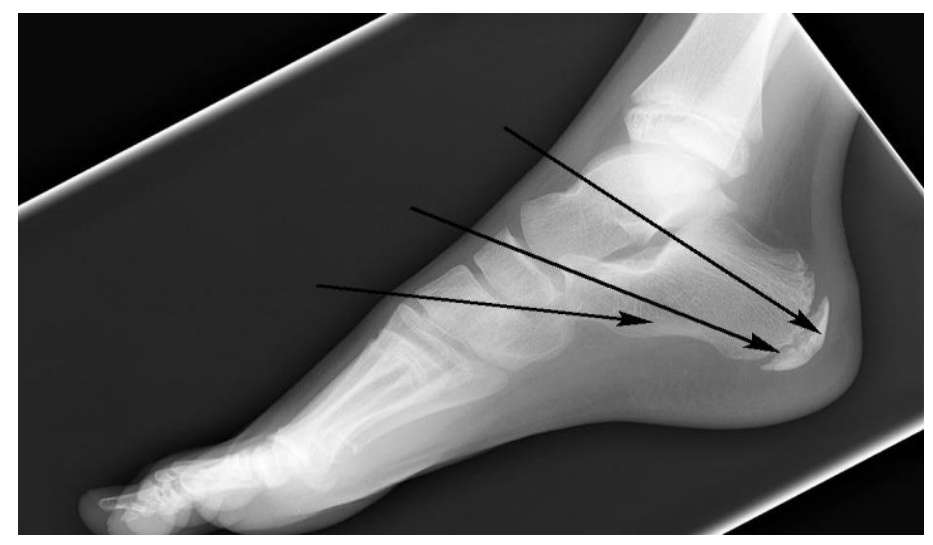

Fig. (2) Apophysis of calcaneus.

\section{Etiology}

With repetitive stress at the growth plate, inflammation develops at the apophysis. High levels of physical activity and obesity in pediatric patients are the main risk factors for Sever's disease. [4]

One study that patients with calcaneal apophysitis were more likely to have a greater body mass index, increased weight, and increased height. There are several theories regarding the cause of calcaneal apophysitis, but all theories concur that the movement of the apophysis relative to the diaphysis (shaft of the bone) causes trauma to the apophyseal tissues with compression or impact forces [5].

Approximately $60 \%$ of the weight-bearing load oc-curs in the rear foot when standing. One study reported that sports activity that causes the highest levels of pain are those with fre-quent running and jumping, such as soccer [6].

Specific sports reported vary from study to study, but most studies involved some type of track and field activities, including soccer. Children who play competitive sports are more likely to have calcaneal apophysitis [6].

\section{Pathomechanics of Sever's Disease}

Many theories have been put forward to explain the formation of Sever's disease, but like other areas of this condition, they have generally been hypothetical or formed on the basis of small populations. Theories may be split into the subheadings of growth and Achilles tendon tightness, biomechanical malalign-ment, trauma, infection, and overweight.

\section{Pathophysiology of Sever's Disease}

The calcaneus is situated at the most plantar posteri-or aspect of the foot. It articulates superiorly with the talus at the subtalar joint and distally with the cuboid, making up part of the midtarsal joint [7].

The Achilles tendon inserts into the lower, posterior aspect of the calcaneus. The plantar fascia originates from the me-dial tubercle on the plantar aspect of the calcaneus. Near the attachment of the Achilles tendon to the cal-caneus, a secondary bony growth center, or epiphysis (Fig. 2), opens in girls aged approximately 5 years and in boys aged 7 to 8 years. It fuses in girls aged ap-proximately 13 years and in boys aged 15 years. Proximal to the epiphysis is the apophysis, where the Achilles tendon actually inserts. The apophysis has its own, slower-growing growth plate, separate fromthe physeal plate [7].

The calcaneal growth plate and apophysis are situated in an area subject to high stress from the plantar fascia and Achilles tendon and may be affected by increased tension on the calcaneus. [7]. 
The main theory in the literature on the pathophysiology of Sever's disease is that it is an overuse syndrome from repetitive microtrauma from an increased traction on the apophysis. This increased traction is believed to be initiated by running and jumping, which causes avulsion fractures on a tiny scale, followed by inflammation. The existence of inflammation in Sever's disease is based upon the radiographic finding that the symptomatic fragmented apophysis is more dense than the asymptomatic apophysis. [8]

\section{Diagnosis of sever's disease}

Pediatric patients between the ages of 8 and 15 years with Sever's injury often complain of heel pain, especially after athletic activities. Patients often report limping or walking on their toes to avoid putting weight on their heels. They often report difficulty running, jumping, or participating in sports activities. These children complain of pain over the posterior heel up to the insertion point of the Achilles tendon. If the cases are intractable, symptoms may be present even at rest. Patients can have complaints in one or both heels [9].

\section{Symptoms}

Patients usually have no history of a specific injury.

- pain in the area of the calcaneal apophysis in an immature athlete

- pain increased with activity or impact

- stretch of the triceps surae exacerbates heel pain

- can display warmth, erythema, \& swelling.

The location of the painful area is variably reported. Sever reported a clinical picture of "tenderness about the posterior aspect of the heel, low down." [10]

This corresponds to the apophyseal area located at the postero plantar aspect of the calcaneus.

Meyerding and Stuck, basing their clinical picture on 21 cases retrospectively reviewed and on many other authors, with no study patient population mentioned, describe the pain as being located posteriorly on the heel, which suggests a high likelihood of Achilles tendinitis or achillobursitis as a diagnosis as opposed to apophysitic pain. [10]
A high activity level has been reported in symptomatic individuals, although it has not been specifically measured.

Many authorshave noted anecdotally that affected children were active and vigorous, and that symptoms often presented at the beginning of the sport season and while the child was undergoing a growth spurt. However, none of these studies measured or reported the children's actual activity levels or evidence for growth spurts. Many of the larger studies sourced their subjects from sports medicine clinics or had authors who were physicians in sports-related disciplines. Data from sports medicine clinics introduce the potential bias that subjects are likely to be active people involved in sport. [10]

Physical examination demonstrates tenderness with palpation and compression at the medial and lateral aspects of the heel. There is typically no erythema or heel swelling. [11]

These patients typically have limited ankle joint dorsiflexion, and physical activity worsens the pain. Clinical diagnosis can be made by performing a "squeeze test" with medial and lateral compression of the heel producing pain with this compres-sion and by excluding other differential diagnoses. [11]

\section{Imaging Modalities as aDiagnostic Tool}

Authors on the basis of no more than their subjective opinions have examined radiographs of children with Sever's disease and found greater density of the apophysis in the affected heels. This trait has been found to be inconclusive as a diagnos-tic tool in repeated studies by radiologists, doctors, and podiatric physicians.

\section{Radiographic Evaluation}

When a pediatric patient presents with in-tense heel pain, plain radiographs of the foot are usually obtained as part of the clinical eval-uation in emergency medicine because it has minimal radiation exposure. Radiographic evidence of Sever's injury had been reported to include 2 findings: increased density and fragmentation of the calcaneal apophysis see Figure (4). [12]

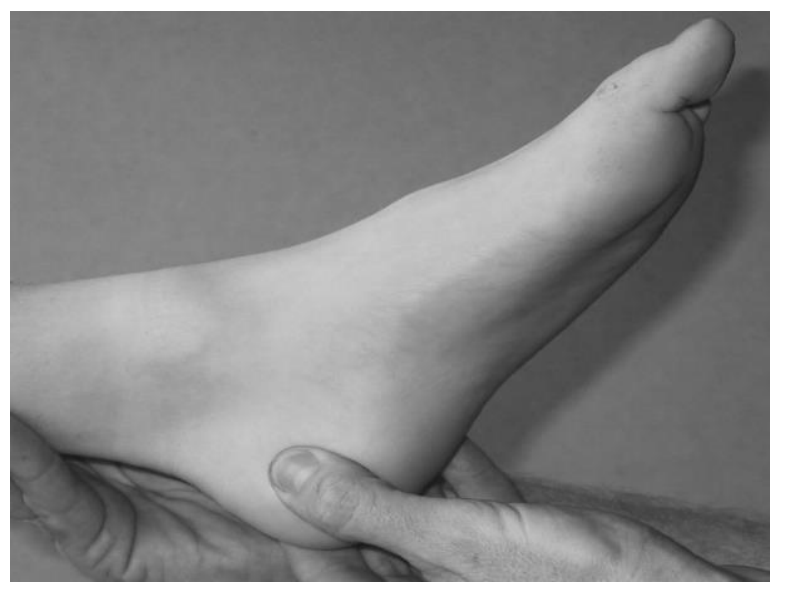

Fig. (3) The squeeze test. Medio-lateral compression of the calcaneal growth plate to elicit pain in Sever's disease[11] 


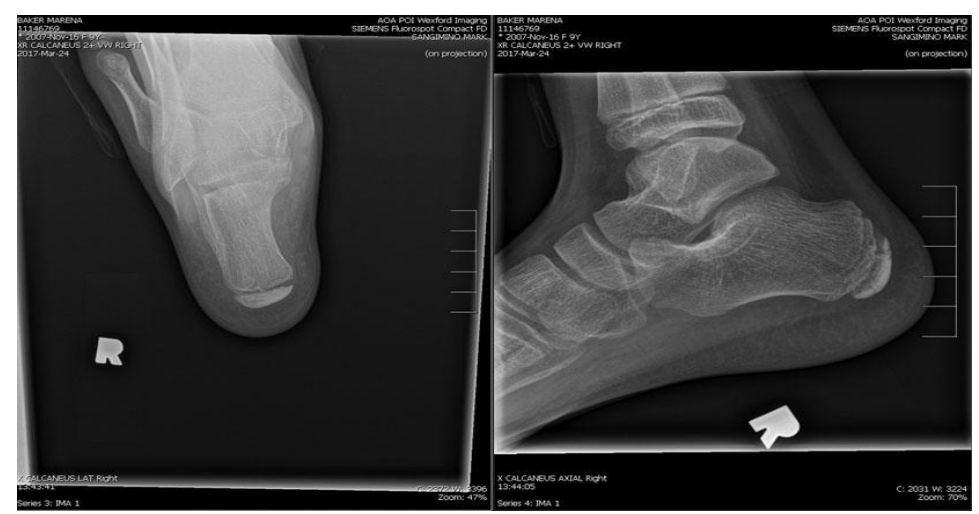

Fig. (4) X-ray of radiographic findings found in Sever's injury: increased density and mild fragmentation of the calcaneal apophysis. [12]

The diagnosis of Sever's disease can be made on clinical ex-amination, and radiographic films may not always be unnecessary. Radiographic plain films should be performed to rule out other causes of heel pain, which can occur in pedi-atric patients such as fractures, dislocations, bone cyst in the calcaneus, osteoid osteoma, or conditions of the tarsal bone. [12] Treatment

Numerous articles have described various types of -treatments for heel pain in children, including stretching, strengthening exercises, limiting physical activity and sports participation, ice, heel lifts for removal of tensile forces on the -calcaneus, correction of malalignment through orthotic use, and even antiinflammatory drugs. However, none of these have been evaluated in prospective treatment trials with validated outcome measures[12]

Other more unusual treatments mentioned- in the literature include avoidance of walking barefoot,- and use of night splints, a cast, and/or crutches. [9]

Most of the recommendations are based on the clinical experience of the authors, or are just a summary of what previous studies have recommended. We have found no prospective randomized clinical trials comparing various treatments for this patient population [9]

In retrospective studies, the authors report on the success of treatment; however, the treatments are mostly generally described, including strengthening, stretching, orthotics, and activity -modifications, with minimal information -regarding outcomes using these measures. [9]

\section{Minimising the inflammation process}

The use of ice, stretching and rest or restriction of activities has been incorporated in the majority of studies. These were commonly provided, as a cluster of treatments and the individual effectiveness of each modality have not been examined within the literature. Even when provided as a cluster of treatments, none of the studies identified in this review reported the results of the effectiveness of this treatment on pain or physical activity levels.
Non-steroidal anti-inflammatory drugs (NSAIDS)

There was one study within the review that incorporated the use of NSAIDS for this condition. This article reported the effectiveness of a topical NSAIDS in the re-duction of pain in a single case report. While the use of this particular topical NSAIDS, Ketroprofen, was contra-indicated in children under the age of 12 [11].

\section{Ice/Activity Restriction}

Ice and activity restriction have been inte-grated into studies for treatment of Sever's disease. Because this pain is highly associ-ated with activity and sports, modification or decreasing physical activities during painful episodes is recommended. These two interventions are used in combination with each other to decrease the inflammatory process in the calcaneal apophysis. Most often, they are the first line of treatment [13].

\section{Stretching/Exercises}

Stretching of the calf muscle is suggested as a treatment because it provides traction directly on the Achilles tendon, which could cause tension on the Achilles and the heel. During rapid periods of growth, the gastrocnemius muscle and Achilles tendon may not always stretch as fast as the bone grows; therefore, the calf muscle pulls on the Achilles at the cal-caneal insertion site, causing pain. Stretching the calf muscle can help alleviate this discom-fort. Exercises can help with the strengthen-ing of specific muscles to decrease the work of the Achilles tendon [13].

\section{Modifying biomechanical factors \\ Immobilization}

Booting or casting for immobilization may oc-cur on an occasional basis for severe cases or for children who are noncompliant with treat-ment. This treatment forces the heel to remain in a resting position in order to reduce inflam-mation and pain. Immobilization devices are usually worn for about 2-4 weeks, and used in conjunction with rehabilitation therapies. [13]. Taping

Taping of the heel and arch of the foot can be done for children such as gymnasts, who can-not wear orthotics during their activity. Many times Sever's pain is associated with excessive pronation of the foot 
and poor arch support. Taping the foot around the arch and heel area has been noted to reduce pain caused by Sever's disease. Arch taping has been shown to have an impact in decreasing pain associ-ated with Sever's and ambulation.

\section{Tape application}

Kinesio taping was applied using the mechanical correc-tion method recommended by Kenzo Kase for calcaneal apophysitis; as such, a 4-6-in. tape portion was cut and pulled from both ends to apply moderate to severe ten-sion (50-75\%) in a way to intersect the insertion point of the Achilles tendon to the calcaneus perpendicularly. The knee was placed in extension and the ankle in max-imum dorsiflexion during the application (Fig. 4) [13].

Arch taping as described by Hunt et al. (82) was used in this case series. Multi-purpose polyethylene coated cloth tape (47 mm, Tyco, Norwood, MA) with natural rubber based adhesive was applied with the patient seated and the involved side's ankle resting on the uninvolved knee. The ankle was maintained in $0^{\circ}$ dorsiflexion during tape application. This taping technique places the main supporting strap across the posterior calcaneus and then in line with the bands of the plantar fascia to anchor just distal to the first through fifth metatarsal heads.

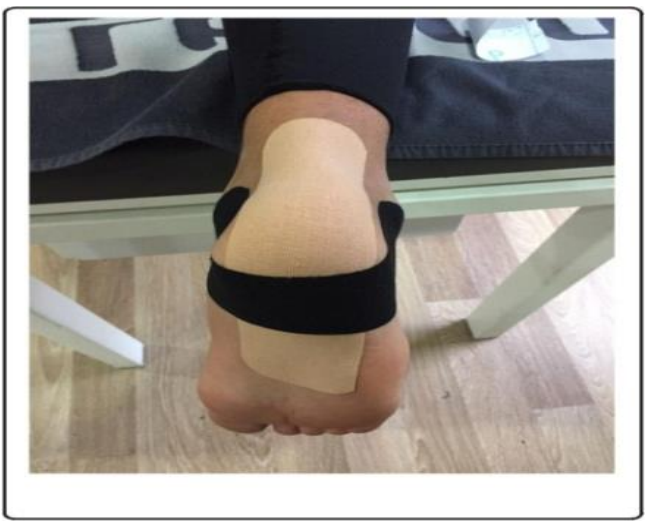

\subsection{Taping}

Fig. (5) Kinesio taping treatment applied to the Achilles tendon and heel region. [13].
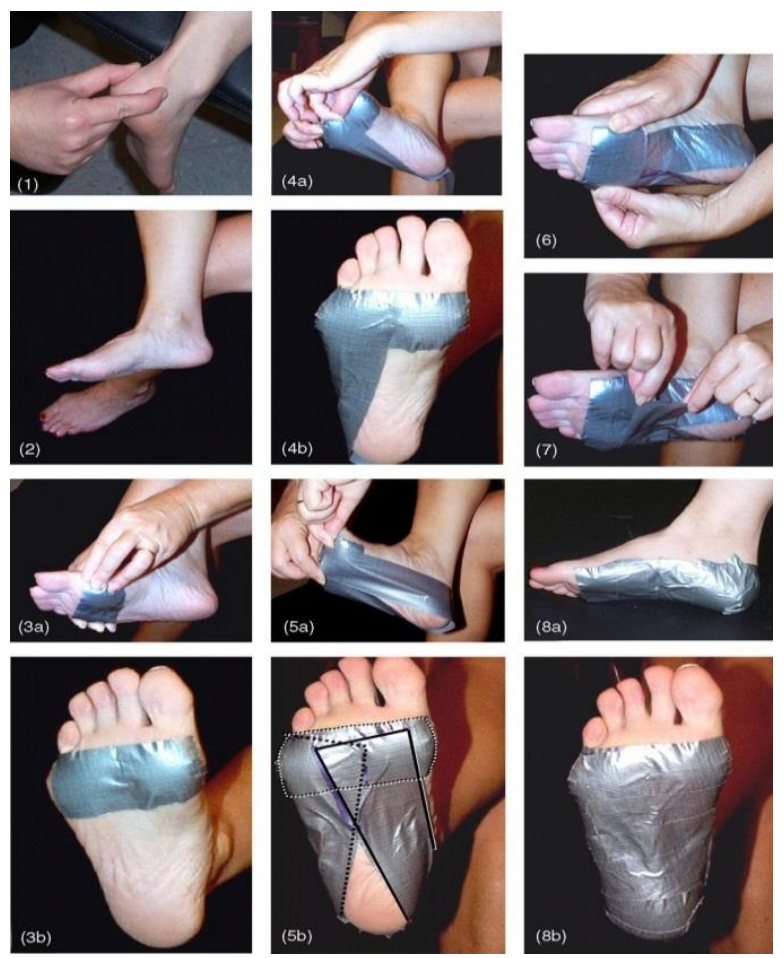

Fig. (6) Pain produced with calcaneal Squeeze test. 2 Place involved foot on opposite knee. Hold ankle in about $0^{\circ} .3$ ( $a$ and b) Apply tape strip that will cover metatarsal heads. 4 ( $a$ and b) Apply long tape-strip to wrap around back of calcaneus and onto lateral plantar foot from approximately the 5th to 3rd metatarsal heads. 5 (a and b) Attach the medial portion of the long tape-strip to approximately the 1st to 3rd metatarsal heads. 6 Apply short tape strip that will cover the metatarsal heads to anchor the long strip. 7 Continue to apply short strips proximally to cover the entire plantar foot. 8 ( $\mathrm{a}$ and $\mathrm{b}$ ) Completed arch taping procedure viewed from medial and plantar surfaces. [14] 


\section{Heel Rise}

Heel rise has been proven to decrease pain associated with Sever's disease. Extra cushion on the heel helps absorb the impact of the ground during jumping or running [13]

\section{Orthoses}

The use of orthotics has been reported within a number of studies and for the purposes of this review, all of the devices that were custom made, moulded around the heel, with or without an arch support are termed as orthoses. There were five publications identified in this review, which examined the efficacy of orth-oses either in comparison to heel lifts or no intervention. Two of these described retrospective case note reviews, while three other publications described two randomised control trials. [13]

\section{Heel lifts}

Many researches have reported the use of heel lifts. All studies have shown heel lifts have reduced pain, but many have utilised them in conjunction with other therapy methods like stretching and cold and have failed to provide findings on the effectiveness of heel lifts on their own. [15]

Modification of activity and return to sport

Because heel discomfort in young athletes is due to increased activity, such as that at the start of the sports season, it is frequently advised to modify some type of exercise. The degree of discomfort and symptoms are frequently used as a guide to establish the appropriate level of involvement in activities [15] injury prevention and reinjury

The identification and adjustment of internal and external risk variables, regardless of a patient's age, are essential to the effectiveness of prevention and treatment of overuse injuries. There is a vacuum in the study literature which has examined the risk factors for the development of post-heel and tendon pain in young athletes in the future. Therefore, we are now unable to establish whether pain/injury causes the potential weakness and reduced movement observed or causes the development of pain/injury. [16]

It is difficult to give a precise suggestion for a preventive fitness programmer. Since heel and tendon discomfort frequently develop in conjunction with greater training, a debate is needed about how young athletes should exercise. Young athletes' maturity and physical size may differ significantly, while they are of the same age. What is a suitable amount of training for one athlete may be too high for another, which increases the danger of overuse. [16].

The monitoring of the symptoms and discomfort of the individual athlete is thus very important when returning to training in this patient group. In collaboration with parents and coaches, a step-by-step instruction on the frequency, intensity and kind of exercise must be customized for each patient. Future research on heel and Achilles tendon pain risk factors in young athletes are very important and are required before precise suggestions can be made. [16].

\section{Discussion}

The results of this review may assist to clarify the causal mechanism of calcaneal apophysitis. Calcaneal apophysitis is generally recognised to be an autonomous disease linked to physiological changes in the calcaneum apophysis as children go through puberty.

Given the causal processes of calcaneal apophysitis, it was not unexpected to discover a range of therapeutic methods in the literature. The treatment choices and effectiveness described in the literature may be based on a variety of hypotheses about causal causes.

Many academics and doctors are still supporting the same basic treatments like ice and sports restriction. The efficacy of additional treatment options such as taping, heel lifts and orthopathy may be predicated on the assumption that calcaneal apophysitis was either caused by greater traction at the calcaneal apophysis or by higher impacts on the planted surface of the heel.

The fast development of adolescents may be a reason for the increase in tension or shortening of the AC hilles tendon. Such a soft tissue shift may potentially lead to an intermediate strain or traction on apophysis when inserted, thus a single heel raise has been called for to minimise this strain when foot wearis are worn.

Sever's disease may usually be diagnosed by a major complaint of heel pain, clinical sessment, and $\mathrm{X}$-ray radiography. [17]. Treatment methods rely on pain, quality of life impact, research indicates that children who are doing sports are more likely than others to have Sever disease, future studies on longterm impacts and complications may help and potentially enhance their immediate treatment strategies.

\section{Conclusion}

The most obvious sign of severe illness is heel discomfort, typically exacerbated by physical activity like walking, jogging, or skipping. Sometimes the discomfort may be so intense that it limps and affects physical performance in athletics. Sever's disease is most often clinically diagnosed and many doctors feel that radiographic assessment is unnecessary, however if calcaneal apophysitis is not identified without $\mathrm{x}$ rays, an injury needing more severe treatment may be overlooked. Edematous alterations in calcaneal apophysis may occur via MRI, which may spread to neighbouring calcaneal tuberosity, The research indicates that most people with the Sever illness are able to return to sport in a few weeks to two months with appropriate management.

\section{References}

[1] A. M. James, C. M. Williams, and T. P. Haines, "Health related quality of life of children with calcaneal apophysitis: child \& 
parent perceptions," Health Qual. Life Outcomes, vol. 14, pp. 1-7, 2016.

[2] P.Tu and J.R.Bytomski, "Diagnosis of heel pain," Am. Fam. Physician, vol. 84, pp. 909916, 2011.

[3] R. J. Price, R. D. Hawkins, M. A. Hulse, and A. Hodson, "The Football Association medical research programme: an audit of injuries in academy youth football," Br. J. Sports Med., vol. 38, pp. 466-471, 2004.

[4] H.Chang, S.-S.Kwon, and K.-W.Minn, "Lateral calcaneal artery as a recipient pedicle for microsurgical foot reconstruction," J. Plast. Reconstr. aesthetic Surg., vol. 63, pp. 1860-1864, 2010.

[5] C.Bibbo, D.A.Ehrlich, H.M.L.Nguyen, L.S.Levin, and S.J.Kovach, "Low wound complication rates for the lateral extensile approach for calcaneal ORIF when the lateral calcaneal artery is patent," Foot ankle Int., vol. 35, pp. 650-656, 2014.

[6] C.E.Attinger, K. K.Evans, E. Bulan, P.Blume, and P.Cooper, "Angiosomes of the foot and ankle and clinical implications for limb salvage: reconstruction, incisions, and revascularization," Plast. Reconstr. Surg., vol. 117, pp. 261S-293S, 2006.

[7] R. W. Scharfbillig, S. Jones, and S. D. Scutter, "Sever's disease: what does the literature really tell us?," J. Am. Podiatr. Med. Assoc., vol. 98, pp. 212-223, 2008.

[8] G.C.Hunt, T.Stowell, G.M.Alnwick, and S. Evans, "Arch taping as a symptomatic treatment in patients with Sever's disease: a multiple case series," foot, vol. 17, pp. 178183, 2007.

[9] S. Basra, "Practical pointers on treating Sever's Disease in young athletes," Pod. Today, vol. 24, pp. 72-73, 2011.
[10] G. A. Malanga and J. A. Ramirez-Del Toro, "Common injuries of the foot and ankle in the child and adolescent athlete," Phys. Med. Rehabil. Clin. N. Am., vol. 19, pp. 347-371, 2008.

[11] B. Hosgoren, A. Koktener, and G. Dilmen, "Ultrasonography of the calcaneus in Sever's disease," Indian Pediatr., vol. 42, p. 801, 2005.

[12] O. Kose, M. Celiktas, S. Yigit, and B. Kisin, "Can we make a diagnosis with radiographic examination alone in calcaneal apophysitis (Sever's disease)?," J. Pediatr. Orthop. B, vol. 19, pp. 396-398, 2010.

[13] M.P.McHugh and C.H.Cosgrave, "To stretch or not to stretch: the role of stretching in injury prevention and performance," Scand. J. Med. Sci. Sports, vol. 20, pp. 169-181, 2010.

[14] P.Haglund, "Concerning Some Rare But Important Surgical Injuries Brought On By Violent Exercise.," Lancet, vol. 172, pp. 12$15,1908$.

[15] R. Howard, "Diagnosing and treating Sever's disease in children," Emerg. Nurse, vol. 22, 2014.

[16] G.C.Hunt, T.Sneed, H.Hamann,andS.Chisam, "Biomechanical and histiological considerations for development of plantar fasciitis and evaluation of arch taping as a treatment option to control associated plantar heel pain: a single-subject design," Foot, vol. 14, pp. 147-153, 2004.

[17] D.R.Ramponi and C. Baker, "Sever's Disease (Calcaneal Apophysitis)," Adv. Emerg. Nurs. J., vol. 41, pp. 10-14, 2019. 